\title{
Alteración del estado mental, reporte de un caso
}

Sandra Marcela Monroy Pereira ${ }^{1}$; Aníbal Teherán Valderrama ${ }^{2}$

1 Residente de primer año Medicina de Emergencias. Universidad del RosarioFSFB

2 Docente-Investigador Grupo Complexus Fundación Universitaria Juan N. Corpas

Correspondencia: Dra. Sandra Marcela Monroy, smarcelamonroy@yahoo.com

Como citar: Monroy SM, Teherán A. Alteración del estado mental, reporte de un caso. Cuarzo 2016; 22 (1): 66-72.

Recibido: 14 de marzo de 2016. Aceptado: 23 de mayo de 2016. Publicado: 30 de junio de 2016.

Licencia creative commons (c) (1) ()

\section{Resumen}

La alteración del estado de conciencia es una situación que puede corresponder a múltiples entidades, el diagnóstico y por ende el manejo correcto depende del adecuado abordaje que se le dé al paciente que se presenta al servicio de urgencias con esta alteración. A continuación, se presenta un caso clínico de un paciente de 37 años que es llevado por un familiar al servicio de urgencias por un cuadro de deterioro en el estado de conciencia, asociado a distermias, alucinaciones visuales y auditivas, temblor generalizado, marcado rechazo a la vía oral, tos con expectoración hemoptoica, con antecedente de consumo pesado de alcohol. Al ingreso paciente en mal estado general, taquicárdico, polipnéico, hipertenso, febril, deshidratado, con IMC bajo, a nivel neurológico desorientado, con actitud alucinatoria, temblor de predominio axial, por lo que como primera posibilidad se consideró síndrome de abstinencia por alcohol, con delirium tremens, se sospechó posible tuberculosis pulmonar, por lo que se inició manejo con benzodiacepinas, agonista $\alpha 2$ y tiamina, con mejoría únicamente en valores de cifras tensionales y disminución del temblor, sin embargo persiste con actividad alucinatoria y fluctuación en el estado de conciencia, se tomaron múltiples paraclínicos, los cuales mostraron un líquido cefalorraquídeo sugestivo de meningitis bacteriana en el que también se detectó bacilo tuberculoso, por lo que se inició cubrimiento antibiótico de amplio espectro que luego se escalonó según aislamiento y antibiograma, también se inició tetraconjugado para tuberculosis meníngea, con mejoría de los síntomas de ingreso, sin secuelas neurológicas.

CONCLUSIÓN: un abordaje adecuado en el servicio de emergencias del paciente que ingresa con alteración del estado mental, es la guía más importante para poder realizar un diagnóstico certero y así instaurar un tratamiento adecuado y efectivo.

PALABRAS CLAVE: alteración del estado mental, delirium tremens, neuroinfección. 


\section{Alteration of mental state, a case report}

The altered state of consciousness is an issue that can be related with several entities, diagnosis and therefore the appropriate handling relays on the adequate approach to the patient who comes to ER services with such alteration. Here is a clinical case of a 37 patient who was indicted to ER services by a relative with deteriorate state of consciousness symptoms associated with dysthermias, visual and auditory hallucinations, general shaking, strong orally rejection, coughing with hemoptoic expectoration, with alcohol consumption background. At the entry he showed general physical discomfort, tachycardia, polypneic, hypertensive, febrile, dehydrated, with low BMI; in neurological level, disoriented with hallucinatory attitude, predominance axial shivering. Therefore, the first possibility was considered as alcohol withdrawal symptoms with delirium tremens. Pulmonary tuberculosis was considered so it was necessary benzodiazepine treatment including agonist 2 and thiamine. He showed improvement only in blood tension figures and shaking reduction. However, hallucinatory activities and state of consciousness fluctuations persist. Complementary tests were taken and they showed cerebrospinal fluid which suggest bacterial meningitis as well as tubercle bacillus so that a spread spectrum antibiotic cover was started and staggered according to isolation and antimicrobial susceptibility testing. Also meningeal tuberculosis tetra conjugate was made and it showed improvement of the beginning symptoms without neurological effects.

CONCLUSION: An appropriate approach to the patient that comes to ER services with mental state alteration, is the most important guide in order to make an accurate diagnosis and start an adequate and effective treatment.

KEYWORDS: Altered mental status, delirium tremens, neuroinfection.

\section{Introducción}

La alteración del estado mental es un motivo de consulta común en los servicios de emergencias, cerca del $40-60 \%$ de adultos mayores ingresa a urgencias en Estados Unidos por esta condición, sin embargo la población joven puede también ingresar con alteración del estado mental y, en algunos casos, puede ser más sencillo la realización del diagnóstico. Estudios desde la década de los noventa han reportado tasas de hospitalización de hasta el $74 \%$ en todas las edades. La mortalidad por esta condición en definitiva es mucho más alta en la población mayor (1).

Un paciente con alteración del estado mental puede presentarse con una amplia gama de niveles de conciencia que puede encontrarse entre el coma y la combatividad y para esto se utilizan diferentes escalas para categorizar así dicha alteración (1). Sin embargo, es la Escala de Coma de Glasgow (GSC) la herramienta más fiable para determinar el nivel de Conciencia en un paciente (2). Los cambios agudos en el estado mental generalmente se desarrollan en lapsos de horas a días y requieren un reconocimiento temprano para realizar intervenciones que disminuyan al máximo la posibilidad de muerte o de secuelas permanentes (1).
Son múltiples las afecciones que pueden cursar con alteración del estado mental, cualquier proceso que disminuya o interfiera con la entrega o utilización de sustrato al cerebro puede alterar la actividad cerebral. Hipertermia o hipotermia, hipertensión o hipotensión, hiperglicemia o hipoglicemia, hipoxia o hipercapnia, concentraciones anormales de electrolitos, acumulación de productos de desecho (2). Dentro de las condiciones que más frecuentemente se asocian con alteración del estado mental se encuentran los procesos infecciosos ya sean originados en el sistema nervioso central o como consecuencia de otros procesos sépticos que producen liberación de mediadores proinflamatorios (citocinas, endotoxinas) e inclusive el estado de choque, enfermedades neurológicas vasculares, ictales, lesiones ocupantes de espacio, alteraciones cardiopulmonares, renales, metabólicas, trauma (especialmente trauma craneal), ingesta de tóxicos que puede ser alcohol o de otra naturaleza (2,3). Es común que los pacientes con alteración del estado mental tengan más de un diagnóstico y en algunos casos no se logra establecer la condición que ocasionó la alteración del estado mental.

La adecuada realización de la historia clínica, incluyendo si el paciente previamente ha presentado episodios de alteración del estado mental, es clave para realizar un diagnóstico adecuado (1). 
El consumo de etanol ha sido aceptado socialmente durante décadas, sin embargo, esto conlleva a un aumento en la presentación de dependencia y adicción a este tóxico. Las consultas al departamento de emergencias relacionadas con consumo de etanol en los adultos mayores son en su mayoría para el manejo de las secuelas por el abuso crónico del etanol, mientras que los adolescentes y adultos jóvenes son tratados principalmente por cuadros de intoxicación aguda. Cuando un paciente con consumo crónico de alcohol ingresa por alteración del estado mental, se debe considerar la presencia de encefalopatía de Wernicke o encefalopatía hepática. En los pacientes alcohólicos que se presentan después del cese o disminución del consumo de etanol se debe evaluar la presencia de signos de abstinencia, con el objetivo de prevenir la progresión a una abstinencia severa o un delirium tremens (3).

\section{Información del paciente}

Edad: 37 años, sexo: masculino, origen étnico: mestizo, profesión: habitante de calle, MC: "esta raro, no se mueve de la cama y esta alucinando". EA: paciente con cuadro de 7 días de alteración en el comportamiento dado por episodios de agresividad, lenguaje incoherente, temblor difuso y actitud alucinatoria, sin presencia de episodios convulsivos, paciente habitante de calle sin adecuada red de apoyo, otros habitantes de calle al verlo mal deciden informar a familiar, quien lo recoge y lo lleva a su casa, donde ha presentado aumento de episodios de agitación, hipertermia, lenguaje no coherente, temblor que limita actividades diarias, emesis con contenido sanguinolento y episodios de hemoptisis, dada ausencia de mejoría decide consultar. Familiar refiere que hace 4 días ha presentado tos con pintas de sangre, arcadas múltiples sin emesis, además de hiporexia, solo ha recibido pequeñas cantidades de sales de rehidratación oral, marcha antálgica, deformidad en miembro inferior derecho, familiar refiere hace 2 días orina colúrica. ANTECEDENTES: Personales: habitante de calle; Patológicos, Farmacológicos, Quirúrgicos, de Hospitalización y Familiares no hay información; no hay claridad de episodios previos de síndrome de abstinencia; Tóxico - alérgicos: consumo de 3 medias de alcohol etílico "chamber" al día, hace más de 10 años, no consumo de otros tóxicos.

\section{Hallazgos clínicos}

Examen físico: peso: $51 \mathrm{~kg}$, talla $168 \mathrm{~cm}, \mathrm{IMC}: 18 \mathrm{~kg} /$ $\mathrm{m} 2$, mal estado general, caquéctico, diaforético, sin difi- cultad respiratoria, no ictericia, no aliento alcohólico, impresiona ansioso al examen físico, intenta retirarse acceso venoso en múltiples ocasiones, no ha requerido inmovilización desde ingreso. Con signos vitales TA: 110/85 mmhg, FC: 124 lpm, FR: 24 rpm, SAT: 89\% FiO2: 0.21, T: $39.1{ }^{\circ} \mathrm{C}$. Normocéfalo, escleras anictericas, conjuntivas normocrómicas, isocoria reactiva $3 \mathrm{~mm}$, orofaringe no eritematosa, mucosas secas, cuello sin adenopatías, no ingurgitación yugular, tórax de expansión simétrica, no dificultad respiratoria o uso de músculos accesorios, ruidos cardiacos rítmicos taquicardicos, no soplos, ruidos respiratorios sin agregados, murmullo vesicular conservado, abdomen no distendido, no doloroso a la palpación profunda o superficial, no signos de irritación peritoneal, no masas o megalias, extremidades simétricas, con presencia de deformidad a nivel de rodilla derecha, sin presencia de calor o eritema, con dolor a la movilización pasiva, no presencia de derrame sinovial, no cianosis, llenado capilar mayor a dos segundos, pulsos simétricos conservados. Neurológico: alerta, desorientado en tiempo, espacio y persona, mirada de extrañeza, actitud alucinatoria, glasgow 12 (o4v2m6), afasia global, disartria severa, no ejecuta órdenes sencillas, juicio comprometido, isocoria normoreactiva, simetría y sensibilidad facial conservadas, no déficit de pares bajos, fuerza conservada en 4 extremidades, normoreflexia, sensibilidad normal, temblor de predominio axial.

\section{Evaluación diagnóstica}

Al ingreso del paciente a urgencias, los cambios más significativos a nivel del exámen físico son su hipertensión que mejora con la administración de clonidina oral, la taquicardia y la fiebre que persisten los primeros días, los cambios de la esfera mental van mejorando lentamente en las primeras dos semanas. En cuanto a los hallazgos paraclínicos el paciente ingresa con un transtorno electrolítico múltiple que requiere corrección por largos periodos de tiempo en especial de la hipokalemia, no presenta alteración en las pruebas de función renal, hemograma solo evidencia aumento en los valores de los volúmenes corpusculares sin otros cambios, hallazgo que permanece sin cambios a lo largo de su estancia hospitalaria, las pruebas de función hepática evidencian desde el ingreso leve hiperbilirrubinemia a expensas de la indirecta, con elevación discreta de transaminasas que no supera el doble del valor superior de referencia, con aumento progresivo de las mismas días posteriores al inicio de manejo con tetraconjugado, por lo que requirió suspensión de di- 
cha terapia y con el retorno a su estado basal se reinició de manera progresiva con mejor tolerancia. Los tiempos de coagulación solo evidencian prolongación leve del ptt, el cual se mantuvo estable durante la estancia hospitalaria. Las imágenes diagnósticas utilizadas en este caso solo evidenciaron infiltrados intersticiales reticulonodulares difusos en la radiografía de torax (Figure 1), en la TACAR de tórax múltiples bulas de predominio apical izquierdo, bronquiectasias, patrón de árbol en yema, consolidaciones subpleurales apicales posteriores de predominio derecho, derrame pleural bilateral de predominio derecho (Figure 2), la tomografía computarizada de craneo simple no mostró lesiones intracraneales agudas, solo cambios leves de atrofia cortical y subcortical (Figure 3).

El paciente cuenta con una red de apoyo débil y es habitante de calle, por lo que a su ingreso no cuenta con aseguramiento en salud, el servicio de trabajo social gestiona su vinculación y se registra como beneficiario del fondo financiero distrital. Dado que es atendido en un hospital de la red pública, este no cuenta con todos los servicios requeridos en especial de laboratorio, por lo que en algunos casos requiere la remisión de las muestras a laboratorios externos, lo cual hace que se demore la confirmación de estos resultados. También, dada la sobreocupación permanente del hospital algunos estudios como la

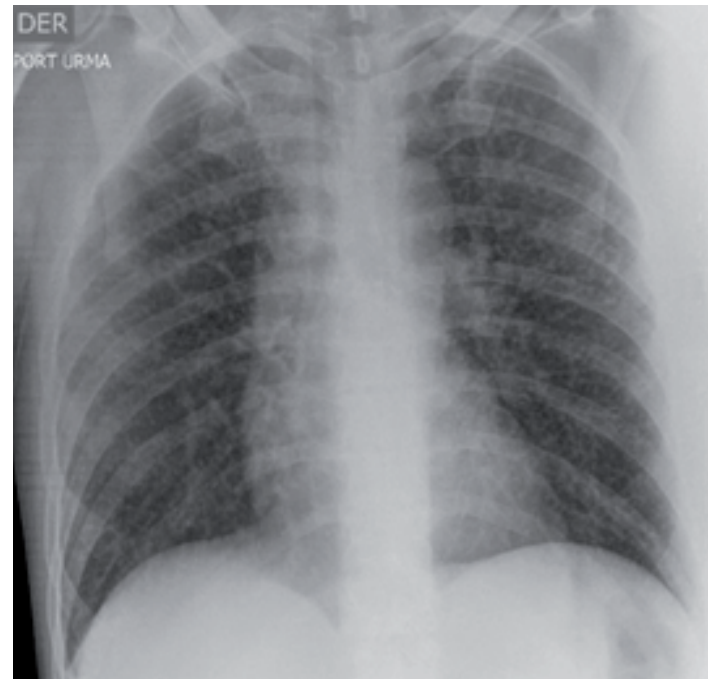

FIGURA 1. Radiografía de tórax con opacidades intersticiales reticulonodulares bilaterales. fibrobroncoscopia y el ecocardiograma se demoran varios días para el agendamiento de la cita y la realización del estudio, retrasando así el diagnóstico y el manejo.

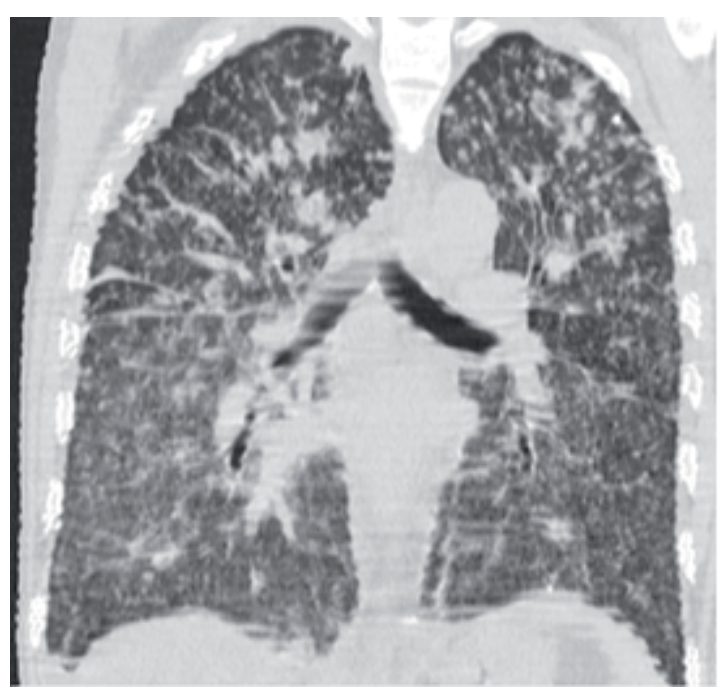

FIGURA 2. TACAR de tórax con múltiples bulas de predominio apical izquierdo, bronquiectasias, patrón de árbol en yema, consolidaciones subpleurales apicales posteriores de predominio derecho, derrame pleural bilateral de predominio derecho.

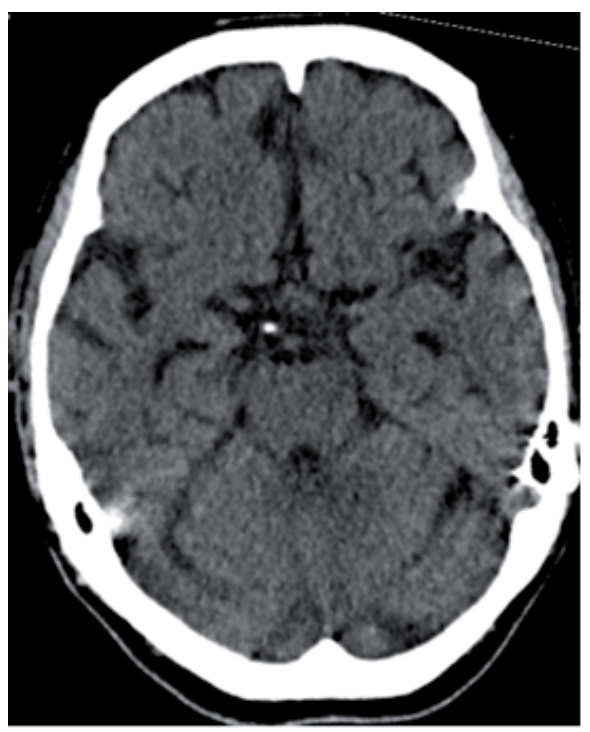

FIGURA 3. Tomografía de cráneo simple con cambios leves de atrofia cortical y subcortical. 
Desde el ingreso del paciente el síntoma mas llamativo fue la alteración del estado mental y dados los antecedentes y los hallazgos del exámen físico de ingreso se consideró como primera posibilidad un síndrome de abstinencia que en la primera valoración obtiene una puntuación de 36 en la escala de CIWA-ar, lo cual indica abstinencia severa y por el curso del cuadro clínico se consideró la presencia de un delirium tremens para lo que se inició manejo médico, además por su estado de deshidratación se consideró trastorno electrolítico concomitante. Por su antecedente de consumo problemático de alcohol también se debía descartar hepatopatía que pudiera desencadenar una encafalopatía, llamaba mucho la atención la presencia de fiebre y síntomas respiratorios, por lo que también se estudió para procesos infecciosos en especial a nivel pulmonar y de sistema nervioso central. Desde el ingreso se sospechó un posible estado de inmunosupresión por lo que se solicitó asesoría pretest y toma de VIH 1 y 2, el cual fue negativo con niveles de CD4 normales.

\section{Intervención terapéutica}

El paciente requirió terapia farmacológica principalmente, desde el ingreso se manejó con bolos de cristaloides para corregir su estado de deshidratación al ingreso con lactato de ringer, posteriormente con cloruro de sodio al $0.9 \%$, luego se continuaron únicamente líquidos basales; para su condición de consumo crónico de alcohol se indicó manejo con tiamina inicialmente $500 \mathrm{mg}$ IV cada 8 horas los primeros 5 días, posteriormente se disminuye a $500 \mathrm{mg}$ IV al día, hacia la segunda semana de estancia hospitalaria se cambia a presentación oral $300 \mathrm{mg} /$ día, ácido fólico desde el ingreso $1 \mathrm{mg}$ oral al día, sulfato de magnesio 1 ampolla al 20\% IV al día durante 4 días.

Como parte del manejo del síndrome de abstinencia se indicó clonidina $75 \mathrm{mcg}$ oral cada 12 horas y lorazepam que se inició a dosis de $12 \mathrm{mg} /$ día, con destete progresivo según indicación de psiquiatría, con dosis al egreso de 3 $\mathrm{mg}$ /día. Por hipokalemia presentada desde el ingreso se indicó reposición con cloruro de potasio IV a $4 \mathrm{meq} / \mathrm{hora}$ durante 3 días, luego se continuó reposición con gluconato de potasio oral $10 \mathrm{cc}$ cada 8 horas, que a los 3 días requiere cambio nuevamente por vía parenteral dada reagudización de hipokalemia durante 2 días adicionales. El proceso infeccioso del sistema nervioso central fue manejado empíricamente con vancomicina 2 gr IV cada 12 horas y ceftriaxona 2 gr IV cada 8 horas, que se inician al día siguiente del ingreso durante 3 días, hasta obtener resultado de cultivo de líquido cefalorraquídeo, posterior

\begin{tabular}{|c|c|c|}
\hline $21 / 02 / 2017$ & Ingreso a urgencias sala de reanimación & Se inicia estudio y manejo para síndrome de abstinencia en sala de reanimación. \\
\hline $22 / 02 / 2017$ & Continúa en sala de reanimación & $\begin{array}{l}\text { Dada mejoría parcial de los síntomas neurológicos de ingreso y teniendo en } \\
\text { cuenta múltiples factores de riesgo se decide realización de punción lumbar, } \\
\text { que por hallazgos de citoquímico y gram sugieren meningitis bacteriana por lo } \\
\text { que se inicia antibiótico de amplio espectro (ceftriaxona más vancomicina). }\end{array}$ \\
\hline 24/02/2017 & & $\begin{array}{l}\text { Por hemomptisis mencionada al ingreso se solicita broncoscopia más } \\
\text { lavado broncoalveolar (negativos para bacilo tuberculoso u otros gérmenes), } \\
\text { cultivo de líquido cefalorraquídeo con crecimiento de Staphylococcus } \\
\text { aureus multisensible por lo que se desescalona antimicrobiano (oxacilina por } \\
14 \text { días). }\end{array}$ \\
\hline $11 / 03 / 2017$ & & $\begin{array}{l}\text { Se inicia corticoide parenteral como complemento de terapia para TB } \\
\text { meníngea, cursa con enfermedad diarreica aguda, se documenta gastroenteritis } \\
\text { de origen parasitario, se inicia cubrimiento con metronidazol oral. }\end{array}$ \\
\hline
\end{tabular}


a esto se desescalona terapia antimicrobiana a oxacilina 2 gr IV cada 4 horas durante 17 días. Desde el punto de vista mental el paciente requiere inicio de haloperidol gotas 10-0-20 por persistencia de agitación psicomotriz y síntomas psicóticos, que se inicia a la semana siguiente de su ingreso, se mantienen dosis sin cambios hasta su egreso. Para el manejo de la tuberculosis meníngea y pulmonar se indica tetraconjugado primera fase (rif 150 , iso 75 , pira 400, etamb 275) 3 tab día, el cual se inició al tercer día de estancia hospitalaria, previa valoración por grupo de $\mathrm{TB}$, ésta terapia debió ser suspendida a los 13 días de iniciado por aumento de las transaminasas, sin embargo al reevaluar el patrón de elevación y normalización de estos paraclínicos consideran posible error de laboratorio dado que las transaminasas disminuyen notablemente antes de suspender la terapia antituberculosa, por lo que deciden reiniciar manejo tetraconjugado y hacer seguimiento de pruebas de función hepática. Se indicó piridoxina $50 \mathrm{mg}$ oral al día la cual se inicia a los 3 días de inicio de isoniazida, dexametasona $16 \mathrm{mg}$ iv cada día, como parte del esquema de tratamiento para TB meníngea, se postergó su inicio hasta completar esquema antimicrobiano para meningitis bacteriana. Heparina de bajo peso molecular (Enoxaparina) $40 \mathrm{mg} \mathrm{sc}$ cada día como profilaxis antitrombótica, se inicia 1 semana después de su ingreso y se mantiene durante toda la estancia hospitalaria. Metronidazol $500 \mathrm{mg}$ vo cada 8 horas por 7 días para enfermedad diarreica aguda parasitaria presentada durante hospitalización.

\section{Seguimiento y resultados}

El paciente durante su estancia hospitalaria presenta mejoría notable de estado mental, sin deterioro de su estado de conciencia, en ocasiones con reaparición de síntomas psicóticos, responde bien a manejo psicofarmacológico instaurado por psiquiatría, no presenta deterioro neurológico ni respiratorio, con resolución de cuadro febril, sin nuevos síntomas de abstinencia por alcohol, pero por su estado de no afiliación a una entidad aseguradora en salud, no es posible hacer seguimiento ambulatorio.

\section{Discusión}

Este reporte de caso representa un reto para el médico de emergencias al afrontar un paciente con alteración del estado mental como principal motivo de consulta, que finalmente es explicado por un sinnúmero de condiciones simultáneas, que deben sospecharse, para poder diagnos- ticarse y tratarse a tiempo y disminuir así las posibles secuelas o los resultados adversos en el paciente.

La alteración del estado mental es un término que tiene varios sinónimos como confusión, alteración del comportamiento, letargo, agitación, psicosis, desorientación, falta de atención y hasta alucinaciones (4). Ésta condición se presenta como consecuencia de múltiples alteraciones a nivel mental y orgánico, que pueden cursar de forma única o simultánea.

El paciente con consumo crónico de alcohol presenta múltiples problemas físicos y psicosociales, que con frecuencia requieren una consulta en el servicio de emergencias $(5,6)$, el espectro de condiciones relacionadas con consumo de alcohol, que conducen a alteración del estado mental incluyen: la intoxicación alcohólica, alteraciones endocrinas en especial las relacionadas con el metabolismo de la glucosa, trastornos electrolíticos (hiponatremia, hipomagnesemia), cetoacidosis alcohólica, encefalopatía alcohólica (6).

Sin embargo, este tipo de pacientes tienen múltiples riesgos para desarrollar otro tipo de patologías que no están en relación directa con el consumo de alcohol y que también pueden generar alteración del estado mental, tal es el caso de la meningitis o meningoencefalitis, que cuando es de origen bacteriano pueden presentar adicionalmente fiebre, cefalea y/o cambios del comportamiento, de no identificarse y tratarse oportunamente, la meningitis bacteriana se asocia con una morbilidad significativa y con tasas de mortalidad de hasta el 27\% (7).

En un país endémico como Colombia, la asociación a otros factores de riesgo como la probreza y la malnutrición, deben ser tenidos en cuenta para sospechar enfermedades como tuberculosis, en este caso el paciente presentaba previamente síntomas respiratorios con posterior confirmación de tuberculosis pulmonar, pero también se documentó compromiso meníngeo, el cual también explica los cambios conductuales y la alteración del estado mental, este es un caso de tuberculosis pulmonar y extrapulmonar en un paciente inmunocompetente. La meningitis tuberculosa no tratada se asocia con una alta frecuencia de secuelas neurológicas y alta mortalidad si no se trata oportunamente (8).

Aunque los pacientes con consumo crónico de alcohol son menos propensos a desarrollar una intoxicación 
aguda, dado que toleran mejor sus efectos, de presentar un cuadro de intoxicación aguda requiere una monitorización estrecha de múltiples parámetros, uno de ellos la evaluación frecuente del nivel de conciencia, cuando un paciente consumidor de alcohol cursa con una alteración del estado mental, en la que puede haber deterioro en el nivel de conciencia o confusión y delirio, de instauración aguda es necesario descartar eventos concomitantes que se puedan ver enmascarados por la ingesta reciente de alcohol; tales como trauma craneoencefálico, eventos cerebrovasculares, sepsis, neuroinfección, enfermedad hepática o cardiaca, también se debe sospechar e indagar el uso de otro tipo de sustancias de abuso (9).

Cuando el consumidor crónico de alcohol disminuye o suspende abruptamente la ingesta de alcohol se presenta el síndrome de abstinencia que puede variar desde leve hasta muy severo, pudiéndose presentar el delirium tremens, que de no manejarse oportuna y adecuadamente puede ser mortal $(5,9)$. Los pacientes que ingresan por complicaciones asociadas a la ingesta de alcohol sea aguda o crónica, suelen presentar alteraciones hidroelectrolíticas que deben ser corregidas de forma oportuna para evitar complicaciones asociadas, e incluso que estas alteraciones perpetúen la alteración del estado mental que presenta el paciente (5).

En este paciente se considera como primera posibilidad un síndrome de abstinencia por alcohol, dado su antecedente de consumo crónico y suspensión reciente del mismo, además que los síntomas y hallazgos al ingreso son compatibles con esta condición, sin embargo ante la persistencia de algunos síntomas y los factores de riesgo conocidos se consideraron otras opciones, lográndose documentar varias patologías que explican ampliamente los cambios agudos presentados por el paciente a su ingreso y que a medida que recibió el tratamiento fueron mejorando paulatinamente.
La responsabilidad principal del médico de emergencias es considerar siempre el peor escenario posible, ante cualquier motivo de consulta $(4,7)$. Para hacer un diagnóstico acertado lo primero que se debe tener en cuenta es la sospecha clínica, que posteriormente con la realización de una historia clínica dirigida pero completa y un examen físico, orienten al médico hacia la causa que está desencadenando los síntomas y así mismo eso definirá el tipo de estudios complementarios y la terapia que se debe instaurar $(7,9)$. El abordaje de este tipo de pacientes necesita destreza, conocimiento y fortaleza por parte del médico de emergencias (5).

\section{Referencias}

1. Aslaner MA, Boz M, Çelik A, Ahmedali A, Eroğlu S, Metin Aksu N, Eroğlu SE. Etiologies and delirium rates of elderly ED patients with acutely altered mental status: a multicenter prospective study. Am J Emerg Med. 2017;35(1):71-76.

2. King D, Avner J. Altered Mental Status. Clin Ped Emerg Med. 2003;4:171-178.

3. Pitzele HZ, Tolia VM. Twenty per hour: altered mental state due to etanol abuse and withdrawal. Clin North Am. 2010;28(3):683705.

4. Han JH, Wilber ST. Altered mental status in older patients in the emergency department. Clin Geriatr Med. 2013;29(1):101-36.

5. Morgan MY. Acute alcohol toxicity and withdrawal in the emergency room and medical admissions unit. Clin Med (Lond). 2015;15(5):486-9.

6. Allison MG, McCurdy MT. Alcoholic metabolic emergencies. Emerg Med Clin North Am. 2014;32(2):293-301.

7. Dorsett M, Liang SY. Diagnosis and Treatment of Central Nervous System Infections in the Emergency Department. Emerg Med Clin North Am. 2016;34(4):917-942.

8. Khanna SR, Kralovic SM, Prakash R. Tuberculous Meningitis in an Immunocompetent Host: A Case Report. Am J Case Rep. 2016;17:977-981

9. Simpson SA, Wilson MP, Nordstrom K. Psychiatric Emergencies for Clinicians: Emergency Department Management of Alcohol Withdrawal. J Emerg Med. 2016;51(3):269-73. 